\title{
Angular Goos-Hänchen Shift Sensor Using Gold Film Enhanced by Surface Plasmon Resonance
}

Cherrie May Olaya ${ }^{1,2}$, Norihiko Hayazawa ${ }^{1,2}$, Nathaniel Hermosa1, Takuo Tanaka ${ }^{1,2,3}$

1 National Institute of Physics, University of the Philippines, Diliman, Quezon City, Philippines

2 Innovative Photon Manipulation Research Team, RIKEN Center for Advanced Photonics, Japan

3 Metamaterials Laboratory, RIKEN Cluster for Pioneering Research, Japan

\section{Supplementary Information}

\section{Reflectivity and phase under $s$-polarized source}
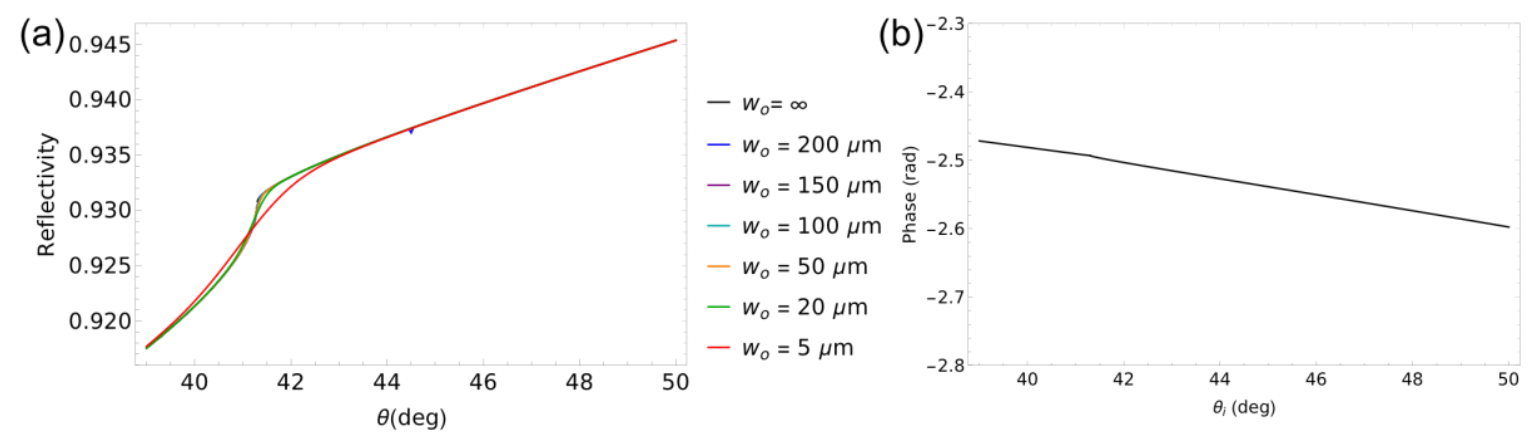

Figure S1: Beam waist dependence of (a) reflectivity and (b) phase under $s$-polarized incident source. No distinct changes are observed as the beam waist is changed. Both the reflectivity and phase values are relatively constant under $s$-polarized illumination. 
FDTD calculation of electric field distribution
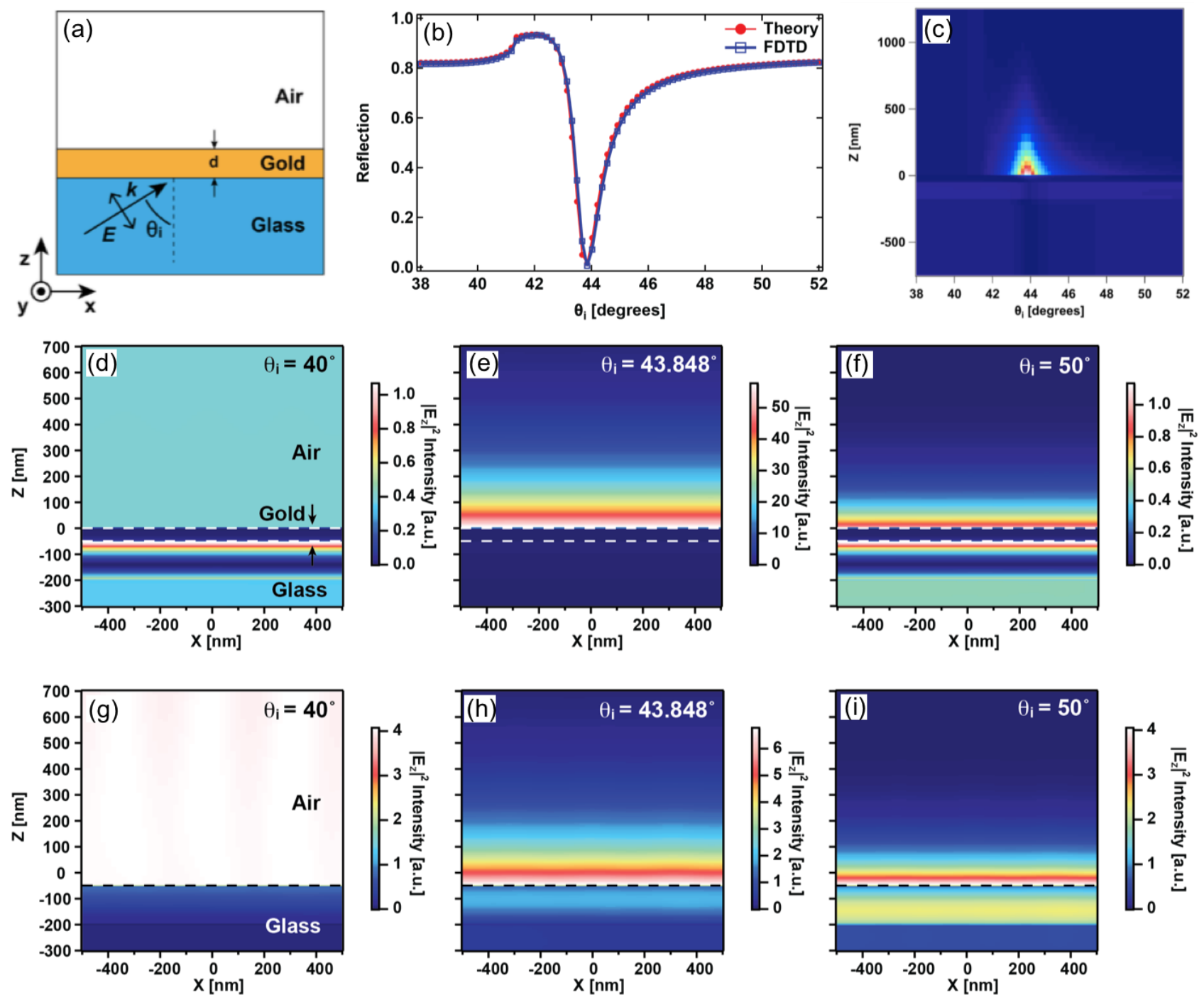

Figure S2: (a) Simulation structure of FDTD calculations performed using Lumerical FDTD. The thickness of the Au film was set at $d=50 \mathrm{~nm}$ for a 632.8-nm $p$-polarized electric field incident at an angle $\theta_{i}$. Bloch boundary condition was used along the $\mathrm{x}$ - and $\mathrm{y}$-axis, and a perfectly matching layer (PML) along the $\mathrm{z}$-axis. The FDTD space was set at $1500 \mathrm{~nm} \times 1500 \mathrm{~nm} \times 1500 \mathrm{~nm}$ with a monitored area of $1 \mu \mathrm{m} \times 1 \mu \mathrm{m}$. Mesh size was set at $20 \mathrm{~nm} / \mathrm{px}$ along the xand $y$-axis and $5 \mathrm{~nm} / \mathrm{px}$ along the z-axis. (b) Comparison of FDTD calculation of reflectivity for different angles of incidence agrees well with theory (see Figure 1a). (c) Electric field at SPR shows an exponential decay along the zaxis. Calculations were performed for (d)-(f) glass-Au-air interface and (g)-(i) glass-air interface. Different angles of incidence were used: (d), (g) incident angle smaller than the SPR angle $\left(\theta_{i}=40^{\circ}\right),(\mathrm{e}),(\mathrm{h}) \operatorname{SPR}$ angle $\left(\theta_{i}=\right.$ $\left.43.848^{\circ}\right)$; and (f), (i) incident angle larger than the SPR angle $\left(\theta_{i}=50^{\circ}\right)$. Strong enhancement of electric field is shown in (e) indicating the excitation of SPP which agrees with theoretical values. 


\section{Incident beam interaction with Au film through hemispherical prism}

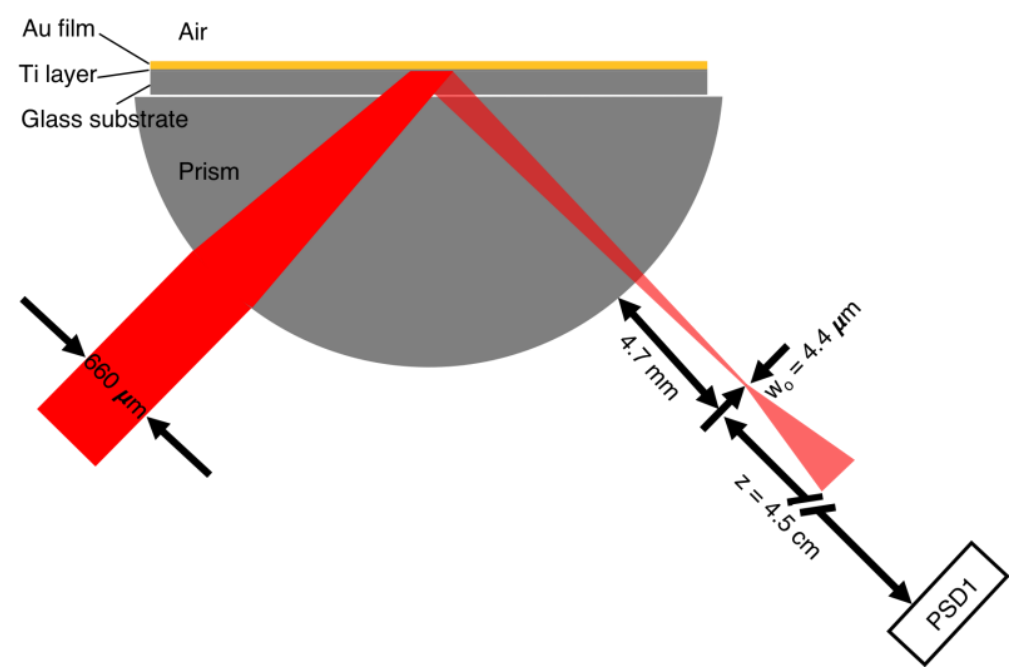

Figure S3: Schematic representation of the interaction of the incident beam as it propagates through the prism and reflects form the Au surface. The incident beam has a $660 \mu \mathrm{m}$ diameter before it impinges the beam. After propagating through the prism, the beam is focused $4.7 \mathrm{~mm}$ from the prism-air interface to beam waist radius of $4.4 \mu \mathrm{m}$.

\section{AFM line profile for total thickness determination}
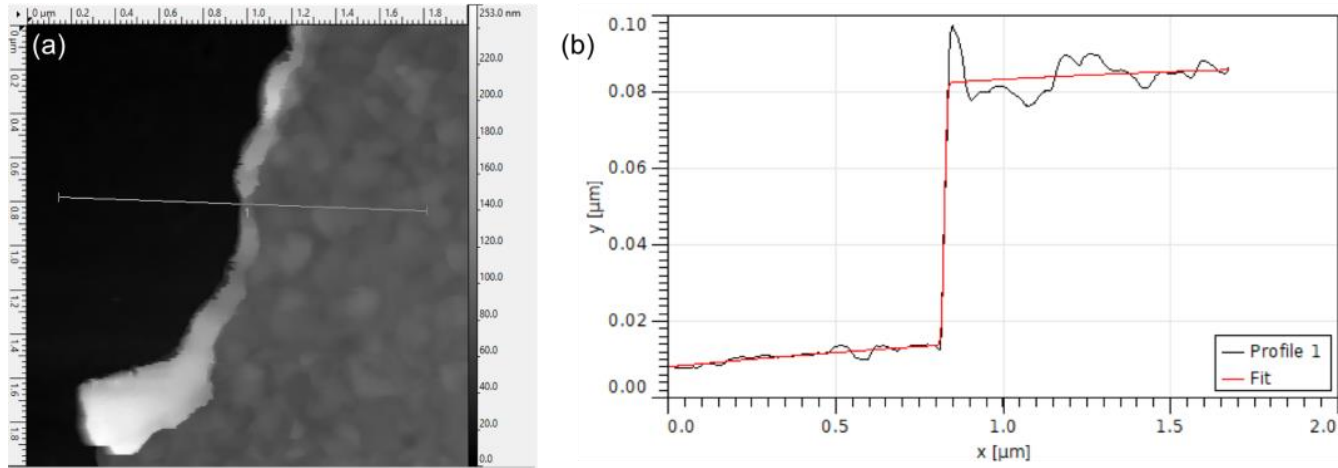

Figure S4: (a) AFM image of the edge of the scratched surface of the Au film and (b) the corresponding line profile for thickness measurement. An edge fitting was performed on the line profile and a thickness of $68.6 \mathrm{~nm}$ was measured. This thickness corresponds to both the Ti layer and the Au film. Additional contribution to the measured thickness could have come from the globular structure of the Au film giving an apparent increase in the actual thickness of the film. 


\section{Reflectivity of hemispherical prism with glass substrate}

(a)
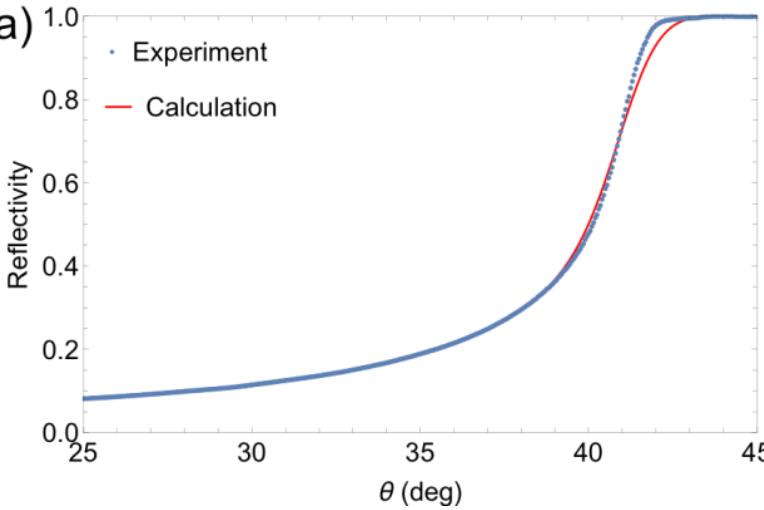

(b)

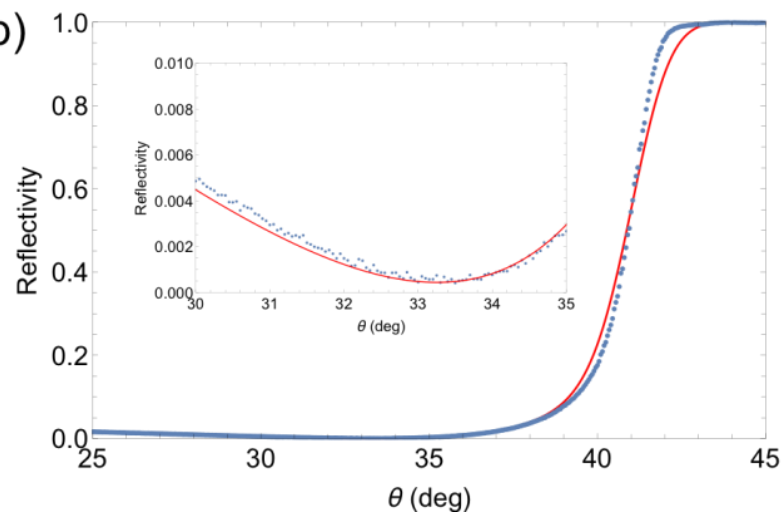

Figure S5: Reflectivity measurement of bare glass slide mounted on the hemispherical prism under (a) $s$ and (b) $p$ polarization states. The matching of the experimental and calculated values show the successful compensation of the $1 \mathrm{~mm}$ polishing of the hemispherical prism to the thickness of the glass slide. Main features of the typical reflectivity plot are shown such as the critical angle and the brewster angle as shown in the inset in (b). Calculations were made using equation 1 of the main text.

\section{Angular Derivative of Reflectivity}

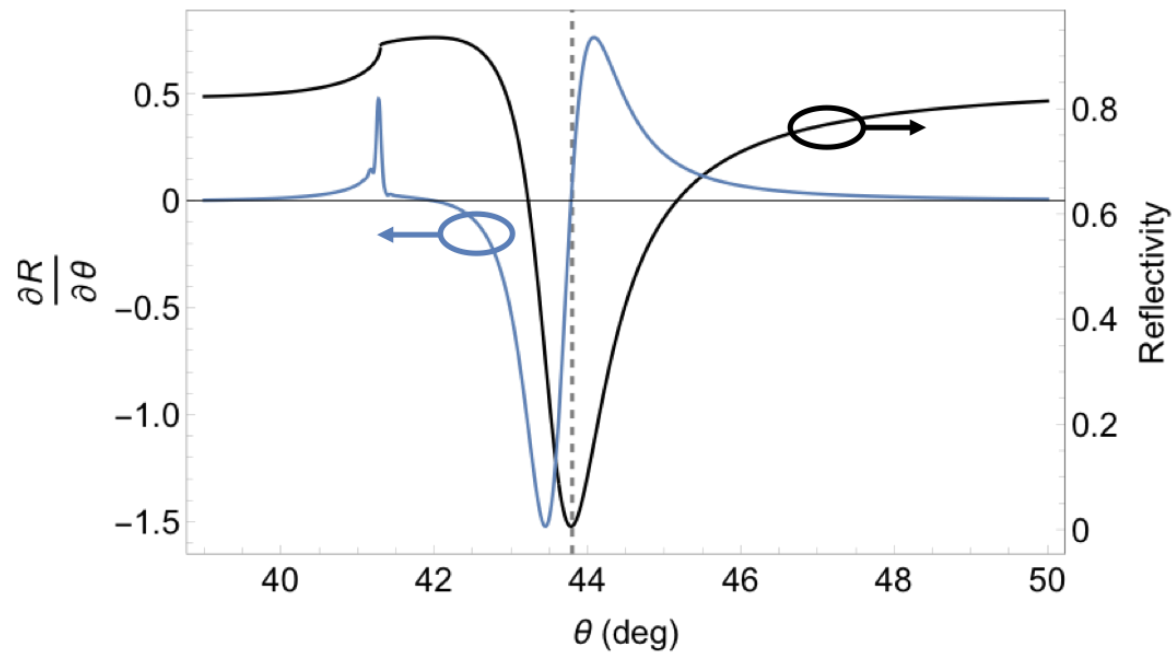

Figure S6: Angular derivative of reflectivity profile (blue) of SPR excitation of Au film. Dashed line shows the location of the SPR angle where $\partial R / \partial \theta$. Black curve corresponds to reflectivity profile for $w_{\bullet}=\infty$ as shown in Figure 1 in the manuscript. 


\section{Position Sensing Device (PSD) Response Measurement}
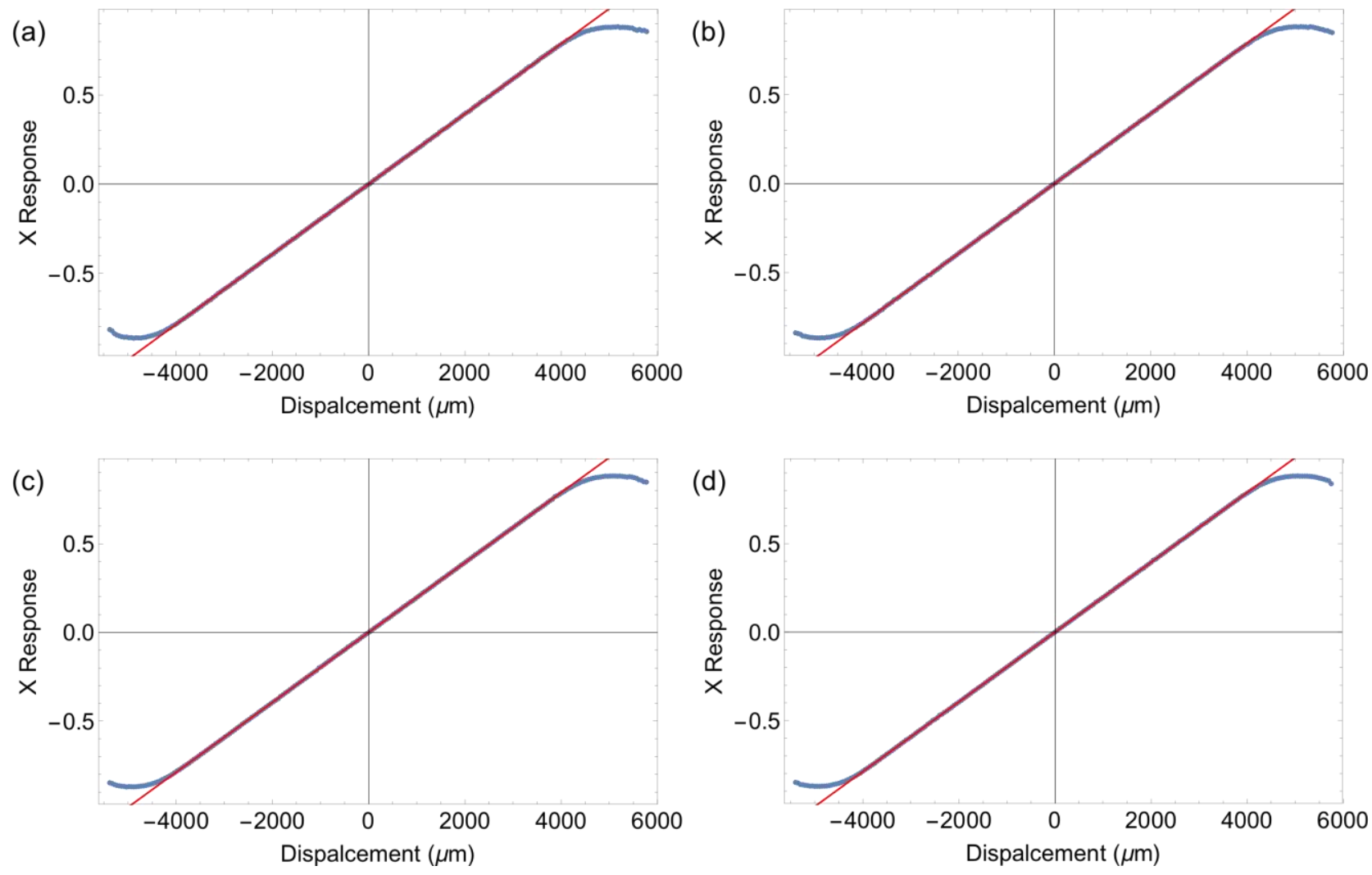

Figure S7: Response of the PSD when a (a) $45.4 \mu \mathrm{W}$, (b) $65.7 \mu \mathrm{W}$, (c) $85.7 \mu \mathrm{W}$, (d) $105.3 \mu \mathrm{W}$ incident laser source scans the horizontal axis of the PSD. Red line shows the linear fitting. Zero response of the PSD is shown at zero displacement which corresponds to the detector center. These measurements were obtained by moving PSD1 using a motorized stage.

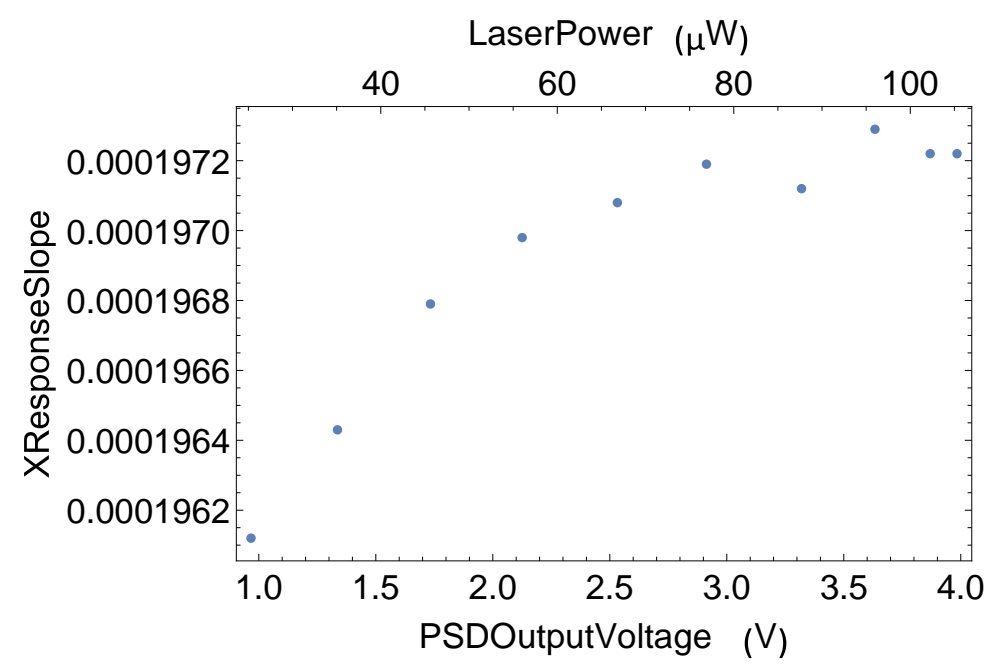

Figure S8: Laser power dependence of PSD response. The slope of response curve shown in Figure S7 determines the conversion of PSD response signal to beam displacement. Very small difference are observed in the slope values with increasing powers. This implies laser power independence of displacement measurement. Values measured agree with the indicated displacement calculation (0.0002) in the PSD manual (Thorlabs). 


\section{EDS Spectra of Au film with and without exhaled breath}

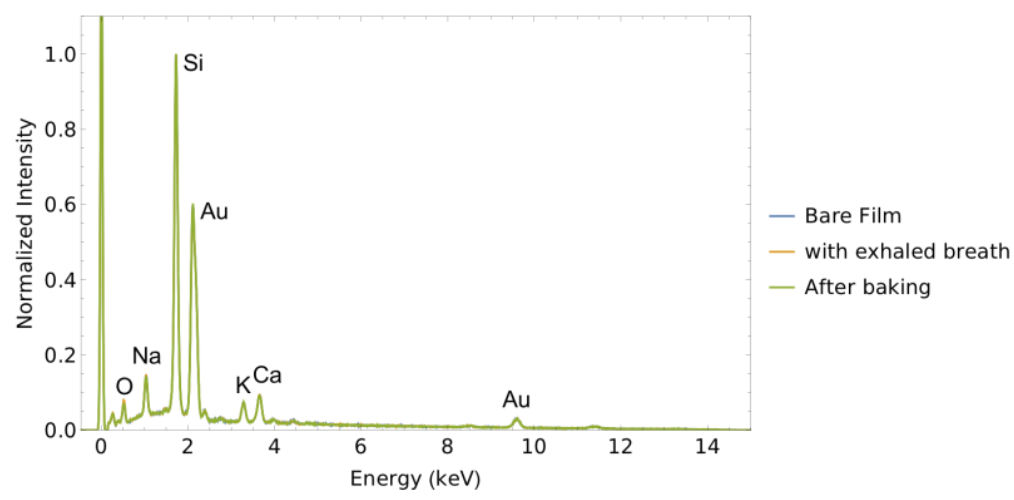

Figure S9: Energy Dispersive X-ray Spectroscopy (EDS) spectrum shows contribution coming from the Au film and the glass substrate and its dopants with dominant peaks labeled in the plot. No distinct difference were observed in the spectrum.

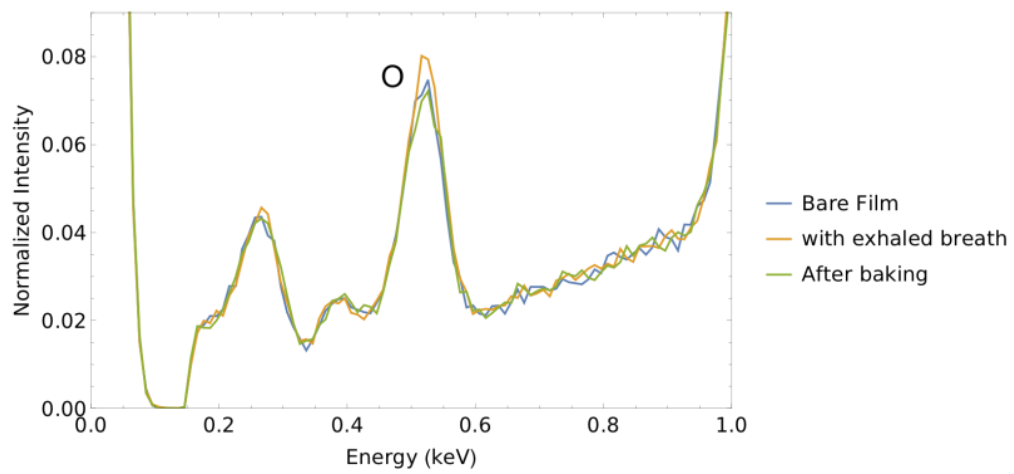

Figure S10: Close inspection of the oxygen peak of the plot shown in Figure S9 show some changes in the 0 peak. Specifically, increase in 0 intensity was shown when the film was exposed to exhaled breath and then decreased after baking. This is indicative of the presence of water molecules that could have been adsorbed on the Au film surface.

\section{Relative Humidity changes over time with exhaled breath}

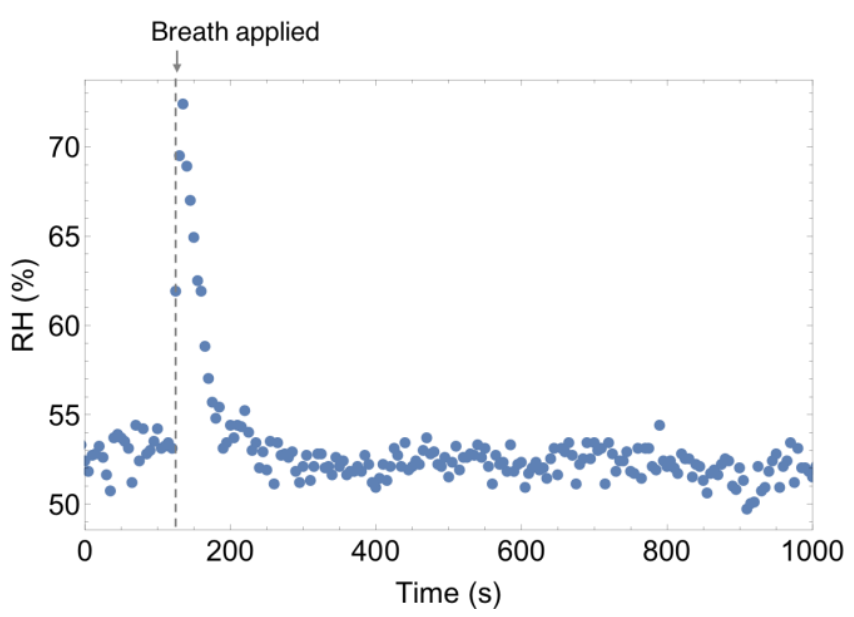

Figure S11: Changes in the relative humidity (RH) over time show that when exposed to an exhaled breath a sudden increase in $\mathrm{RH}$ is observed. After $\sim 2 \mathrm{~min}, \mathrm{RH}$ value goes back to its original value. 